INFLAMMATORY BOWEL DISEASE

\title{
Acute induction of human IL- 8 production by intestinal epithelium triggers neutrophil infiltration without mucosal injury
}

\author{
T Kucharzik, J T Hudson III, A Lügering, J A Abbas, M Bettini, J G Lake, M E Evans, \\ T R Ziegler, D Merlin, J L Madara, I R Williams
}

See end of article for authors' affiliations

Correspondence to: Dr I Williams, Department of Pathology, Emory University, 105D

Whitehead Building, 615 Michael Street, Atlanta, GA 30322, USA; irwilli@ emory.edu

Revised 1 June 2005 Accepted 3 June 2005 Published online first 29 June 2005
Aim: Neutrophil migration in the intestine depends on chemotaxis of neutrophils to CXC chemokines produced by epithelial cells. The goal of this project was to determine if acute induction of a CXC chemokine gradient originating from intestinal epithelial cells is sufficient to induce neutrophil influx into intact intestinal tissue.

Methods and results: The authors developed a double transgenic mouse model with doxycycline induced human IL-8 expression restricted to intestinal epithelial cells. Doxycycline treatment of double transgenic mice for three days resulted in a 50-fold increase in the caecal IL-8 concentration and influx of neutrophils into the lamina propria. Although neutrophils entered the paracellular space between epithelial cells, complete transepithelial migration was not observed. Doxycycline treatment also increased the water content of the caecal and colonic stool, indicating dysfunctional water transport. However, the transmural electrical resistance was not decreased. Neutrophils recruited to the intestinal epithelium did not show evidence of degranulation and the epithelium remained intact as judged by histology.

Conclusions: This conditional transgenic model of chemokine expression provides evidence that acute induction of IL-8 in the intestinal epithelium is sufficient to trigger neutrophil recruitment to the lamina propria, but additional activation signals are needed for full activation and degranulation of neutrophils, mucosal injury, and complete transepithelial migration.
$\mathrm{N}$ eutrophil infiltrates and transepithelial neutrophil migration are among the hallmarks of chronic active inflammatory bowel disease. Current in vitro models of neutrophil transepithelial migration in the intestine postulate that induced secretion of CXC chemokines from the basolateral surface of epithelial cells initiates neutrophil influx by signaling through chemokine receptors on neutrophils. ${ }^{1-3}$ Interleukin-8 (IL-8; designated CXCL8 in current chemokine nomenclature) is a major human CXC chemokine that is rapidly induced in intestinal epithelial cells after stimulation by bacteria and secreted basolaterally. ${ }^{4}$ Epithelial derived IL-8 has been demonstrated to be important to support neutrophil infiltration in various types of human mucosal inflammation including ulcerative colitis ${ }^{56}$ and psoriasis. ${ }^{7}$ Signaling pathways that are involved in neutrophil migration across the mucosal barrier in the intestine have been defined in vitro. ${ }^{8}$

CXC chemokines and the human CXCRl and CXCR2 chemokine receptors are known to play important roles in inflammation associated migration of neutrophils. Neutralising anti-IL-8 antibodies block neutrophil migration across human urothelial cell layers in vitro ${ }^{9}$ and in vivo neutralisation of IL-8 inhibits both neutrophil migration and neutrophil mediated tissue injury. ${ }^{10}$ Mice have a single receptor for CXC chemokines homologous to human CXCR1 and CXCR2. ${ }^{11}{ }^{12}$ This receptor (designated mouse CXCR2) transduces the chemotactic effects of all murine neutrophil attracting CXC chemokines and human IL-8, which has been extensively used as a model chemokine ligand for analysing CXCR2 function in mice. Mice lacking CXCR2 have an increased susceptibility to acute experimental pyelonephritis and decreased migration of neutrophils in a chemical peritonitis model. ${ }^{13}$ Most animal models of inflammatory bowel disease lack infiltration of neutrophils into the mucosa and formation of so-called "crypt abscesses" which is a predominant finding in chronic active human inflammatory bowel disease. Previous transgenic mouse models of CXC chemokine overexpression in intestinal epithelium have been hampered by constitutive expression of the chemokine, ${ }^{14-16}$ resulting in some models showing desensitisation of neutrophils to the effects of constitutively produced chemokine. ${ }^{14}$ The reverse tetracycline transactivator (rtTA) protein permits strong induction of genes linked to a tetracycline responsive promoter following introduction of doxycycline (DOX). ${ }^{17}$ To test the hypothesis that a gradient of a CXC chemokine emanating from intestinal epithelial cells is sufficientindependent of other proinflammatory molecules-to recruit a neutrophil infiltrate into intestinal epithelium, we developed a transgenic mouse model with inducible expression of IL-8 in the intestinal epithelium.

\section{MATERIALS AND METHODS}

\section{Transgenic constructs}

A human IL-8 cDNA with a consensus Kozak translational start site was generated by PCR amplification of pUC-IL- $8^{18}$ with the following primers: 5'-TAGAATTCCACCATGACTTCC AAGCTGG-3' and 5'-TTCTAGAATTATGAATTCTCAGCCC-3'. The PCR product was cloned into pZErO2 (Invitrogen, San Diego, CA, USA) at the EcoRV site and checked by sequencing. The insert was excised with SpeI and XbaI and cloned into the XbaI site of pUHD10-3 ${ }^{19}$ obtained from Dr Herman Bujard (University of Heidelberg, Germany) to create ptetOIL-8. A second tetracycline responsive IL-8

Abbreviations: DOX, doxycycline; $r \mathrm{rtT}$, reverse tetracycline
transactivator; FABP-rTA, Fabp ${ }^{4 X^{\prime}}$ at $-132 / \mathrm{rTTA}$. 
expression vector designated pTRE2IL-8 was prepared by removing the same cDNA from ptetOIL- 8 with BamHI and $\mathrm{XbaI}$ and inserting it into the pTRE2 vector (Clontech, Palo Alto, CA, USA). Transgenic mice were generated by microinjection of either the $1.2 \mathrm{~kb}$ XhoI/BstEII fragment from ptetOIL- 8 or the $2.0 \mathrm{~kb}$ XhoI/AseI fragment from pTRE2IL-8 into $(\mathrm{C} 57 \mathrm{BL} / 6 \mathrm{X} \quad \mathrm{SJL} / \mathrm{J}) \mathrm{F}_{2}$ or $\mathrm{C} 57 \mathrm{BL} / 6$ eggs. Transgenic founders were identified by PCR screening. The tetOIL- 8 construct was detected by PCR amplification of genomic DNA with 5' '-TTGACCTCCATAGAATACACC-3' and 5'CCCTGAACCTGAAACATAAAA-3' yielding a 753 bp amplimer. For the TRE2IL-8 construct, a $220 \mathrm{bp}$ segment of the rabbit $\beta$-globin intron in the construct was amplified using the following primers: 5'-ATCCTGCCTTTCTCTTT-3' and 5'CCACACCAGCCACCACCTTCT-3'.

\section{Mice}

Transgenic mice expressing rtTA only in the distal ileum, caecum, and proximal colon $\left(\mathrm{Fabpl}^{4 \mathrm{X}}\right.$ at ${ }^{-132} / \mathrm{rtTA}$ mice; abbreviated FABP-rtTA) were the kind gift of Dr Jeffrey I Gordon (Washington University, St Louis, MO, USA). ${ }^{20}$ Tetracycline regulated luciferase reporter transgenic mice (tetOluc) were used to characterise the pattern of expression of the FABP-rtTA transgene. ${ }^{21}$ The tetOluc transgene was detected by PCR using 5' GGCGTGTACGGTGGGAGG-3' and 5'-GCAATGTTCCAGGAACCAGGGCG-3' (320 bp amplimer) ${ }^{21}$ In litters of FABP-rtTA mice mated to mice carrying a tetracycline responsive transgene, the presence of the FABP-rtTA transgene was detected by PCR with primers amplifying a $220 \mathrm{bp}$ amplimer (5'-CGCCCAGAAGC

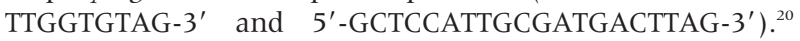
After establishment of a colony of FABP-rtTA and TRE2IL-8 double transgenic mice, double transgenic mice were backcrossed to C57BL/6 mice for four generations. Transgenic mice were induced with DOX by supplementing the drinking
Table 1 Inducibility of IL-8 transgenes in cultured cells from four independent IL-8 transgenic lines*

\begin{tabular}{|c|c|c|c|c|}
\hline Transgenic line & Adenovirus & DOX & IL-8 (ng/ml) & Induction ratio \\
\hline tełOIL-8 \#1 & - & - & 0 & \\
\hline tełOIL-8 \#1 & rtTA & - & 0.3 & \\
\hline tełOIL-8 \#1 & rtTA & + & 2.0 & 7.7 \\
\hline tełOIL-8 \#2 & - & - & 0 & \\
\hline tełOIL-8 \#2 & rtTA & - & 0 & \\
\hline tełOIL-8 \#2 & rtTA & + & 0.1 & Not determined \\
\hline TRE2IL-8 \# 1 & - & - & 0 & \\
\hline TRE2IL-8 \# 1 & rtTA & - & 0.1 & \\
\hline TRE2IL-8 \#1 & rtTA & + & 1.5 & 21.9 \\
\hline TRE2IL-8 \#2 & - & - & 0 & \\
\hline TRE2IL-8 \#2 & rtTA & - & 2.1 & \\
\hline TRE2IL-8 \#2 & rtTA & + & 406.3 & 189.5 \\
\hline
\end{tabular}

*Primary cell cultures (mostly myofibroblasts) were established from the lungs of four independent transgenic lines (two each with either tetOIL-8 or TRE2IL-8 construct). Confluent monolayers of cells were transduced with supernatant containing adenoviruses expressing rtTA. Doxycyline was added to the indicated wells at a final concentration of $2 \mu \mathrm{g} / \mathrm{ml}$. The concentration of IL- 8 in the supernatant three days after addition of the rtTA-expressing adenovirus was determined by ELISA.

tRatio of IL-8 concentration in cells transduced with adenovirus and treated with doxycycline compared to cells only transduced with adenovirus.

water with $0.5 \mathrm{mg} / \mathrm{ml}$ doxycycline $\mathrm{HCl}$ and 5\% sucrose. All experimental protocols employing mice were approved by the Institutional Animal Care and Utilization Committee of Emory University.

\section{Ribonuclease protection assay}

To directly measure rtTA mRNA expression in tissues from FABP-rtTA mice, we performed ribonuclease protection assay on RNA was isolated from tissue using Trizol (Invitrogen). A custom cRNA probe for detecting mRNA

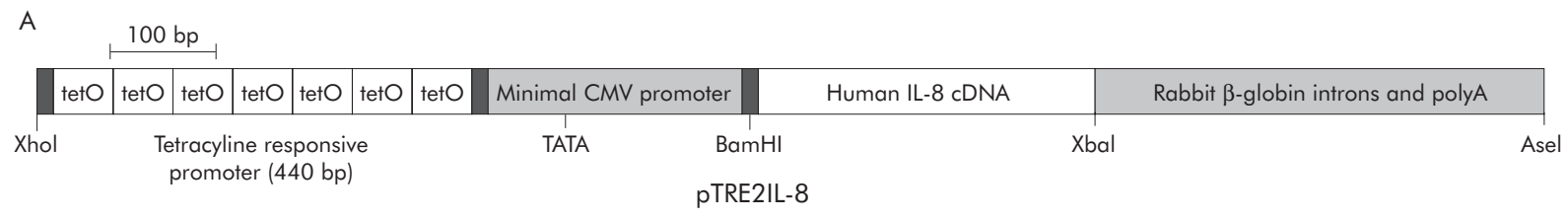
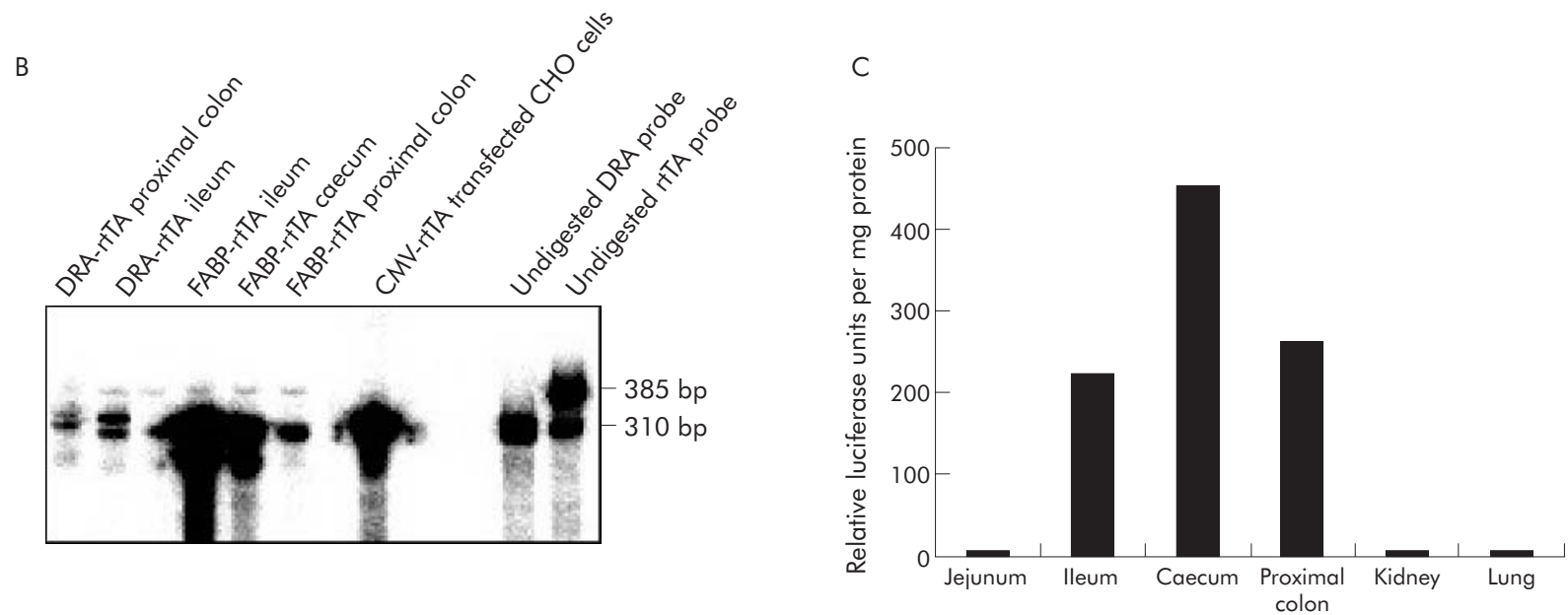

Figure 1 Expression and function of rtTA transgenes in FABP-rtTA and DRA-rTTA transgenic mice. (A) Schematic diagram of the TRE2IL-8 construct. (B) RNA was isolated from tissues of FABP-rtTA and DRA-rtTA mice or a CMV-rtTA transfected cell line. The level of rtTA mRNA was measured by a RNase protection assay in which presence of the rtTA message resulted in protection of a 310 bp band. The highest levels of rtTA mRNA were detected in the ileum and caecum from FABP-rtTA mice. (C) Tissue distribution of induced luciferase activity by DOX in FABP-rtTA $\times$ tetOluc mice. Tissues from FABP-rtTA $\times$ tetOluc double transgenic mice were harvested three days after DOX induction and homogenized. Luciferase activity was determined and normalised to protein concentration. The pattern of luciferase expression in a single animal is shown. A similar pattern was observed in four other double transgenic mice analysed by the same method. 
Table 2 IL-8 levels in tissues of FABP-rtTA $\times$ TRE2IL-8 mice after DOX induction*

\begin{tabular}{lcc}
\hline Tissue & ng IL-8 per $\mathbf{m g}$ total protein & Induction ratiot \\
\hline lleum & 6.4 & 30.8 \\
Caecum & 12.3 & 61.7 \\
Proximal colon & 2.9 & 11.0 \\
Kidney & 0.2 & 2.0 \\
Lung & 0.3 & 1.8 \\
\hline
\end{tabular}

*Tissues were harvested from untreated FABP-rtTA $\times$ TRE2IL-8 mice or double transgenic mice that were given DOX in drinking water for 72 hours. The IL- 8 content of the homogenised tissue was determined by ELISA and normalised to the total protein concentration. The IL-8 values from the DOX-induced mice are shown.

†Ratio of normalised IL-8 concentration in DOX-induced FABP-rtTA $\times$

TRE2IL-8 mice to uninduced double transgenic mice.

transcripts containing rtTA sequences was prepared by subcloning a $308 \mathrm{bp}$ AfeI-HindIII band from rtTA into pBluescript II KS- cut with SmaI and HindIII. In vitro transcription was performed with XhoI linearized plasmid DNA as template, T7 RNA polymerase, and $\alpha-{ }^{32} \mathrm{P}-\mathrm{UTP}$ (Amersham) using the RiboQuant kit (BDPharMingen, San Diego, CA, USA). The 385 bp labeled probe was hybridized overnight at $56^{\circ} \mathrm{C}$ to $20 \mu \mathrm{g}$ of total RNA. The products were digested with RNase A and RNase Tl for one hour at $37^{\circ} \mathrm{C}$. The digested RNA was subjected to electrophoresis through urea/acrylamide gels to allow identification of rtTA mRNA as a 311 bp protected fragment.

\section{Use of recombinant adenovirus encoding rtTA to test DOX inducibility of IL- 8 transgenes}

To test individual transgene integrations in TRE2IL-8 mice and tetOIL- 8 mice, we developed a recombinant adenovirus expressing a modified form of rtTA (KrtTAFFF) with a Kozak consensus translational start site and a modified transactivator containing three minimal activation domains. ${ }^{22}$ KrtTAFFF cDNA was cloned into the Bgl II site in pQBIAdBM5-GFP vector (Qbiogene, Carlsbad, CA, USA). Homologous recombination was done in 293 cells that were co-transfected with vector linearised at the FseI site and long arm DNA from adenovirus 5 lacking the El and E3 genes. GFP positive plaques were selected after 10 days and plaque purified. Supernatants containing recombinant adenovirus were prepared by freeze-thaw lysis of 293 cells three days after adenoviral infection. The inducibility of the individual TRE2IL-8 and tetOIL-8 transgenes was analysed by transducing cultures of myofibroblasts established from neonatal

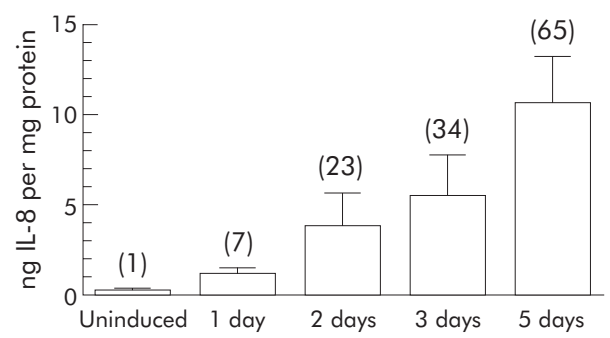

Figure 2 Kinetics of IL-8 induction by DOX in the cecum of FABP-rtTA $x$ TRE2IL-8 mice. FABP-rtTA $\times$ TRE2IL-8 double transgenic mice (two mice per time point) were induced with DOX in their drinking water and the caecum harvested from 1-5 days later. The IL-8 concentrations of tissue homogenates were determined by ELISA and normalised to protein concentration. The induction ratio (ratio of normalised IL- 8 expression in induced double transgenic mice compared with the uninduced mice) is indicated in parentheses above the bar for each time point. A similar time course of DOX induced induction of IL-8 in the caecum was observed in a second experiment.
Table 3 Induction of IL- 8 in serum of FABP-rtTA $x$ TRE2IL-8 mice after DOX treatment*

\begin{tabular}{lcc}
\hline Time & Serum IL-8 $(\mathrm{ng} / \mathrm{ml})$ & Fold induction $v$ baselinet \\
\hline Day 0 & $71(20)$ & 1.0 \\
Day 2 & $266(101)$ & 3.7 \\
Day 5 & $489(47)$ & 6.9 \\
\hline & *Serum IL-8 was measured by ELISA in FABP-rtTA $\times$ TRE2IL-8 mice before \\
treatment with DOX (day 0) or 2 and 5 days after initiation of DOX \\
treatment. The results are expressed as the mean (SD) of three mice per \\
group. A similar degree of IL-8 induction was obtained in two additional \\
experiments of the same type. \\
†The induction ratio is the ratio of serum IL-8 in DOX-induced FABP-rtTA \\
$\times$ TRE2IL-8 mice to uninduced double transgenic mice.
\end{tabular}

lung from each independent transgenic line with recombinant adenovirus encoding rtTA. The IL- 8 content of supernatants from cultures supplemented with DOX $(2.0 \mu \mathrm{g} / \mathrm{ml})$ or receiving no DOX was compared by ELISA. The TRE2IL- 8 transgenic line with the highest induction ratio of IL-8 after administration of DOX was mated to FABP-rtTA mice to establish a colony of double transgenic mice. IL-8 expression in double transgenic FABP-rtTA $\times$ TRE2IL-8 mice was induced by adding DOX $(0.5 \mathrm{mg} / \mathrm{ml})$ to the drinking water.

\section{ELISA for IL-8}

IL-8 was measured by ELISA as previously described. ${ }^{1}$

\section{Luciferase determinations}

Tissues from mice were homogenised in a buffer containing $50 \mathrm{mM} \mathrm{K}_{3} \mathrm{PO}_{4}$ (pH 7.8), $1 \mathrm{mM}$ EDTA, $1 \mathrm{mM}$ DTT, and $10 \%$ glycerol. Aliquots were diluted 1:5 in Reporter Lysis Buffer (Luciferase Assay System, Promega, Madison, WI, USA) and subjected to a single freeze/thaw cycle. After centrifugation ( $14000 \times \mathrm{g}$ for 5 minutes), $100 \mu \mathrm{l}$ of supernatant was added to $100 \mu \mathrm{l}$ of Luciferase Assay Reagent (Promega) and light production measured using a luminometer (Turner BioSystems, Sunnyvale, CA, USA). The results were normalised to protein content of the samples and are reported as relative light units per $\mathrm{mg}$ of protein.

\section{Immunofluorescence staining and confocal microscopy}

Neutrophils were detected in acetone fixed frozen sections using biotinylated anti-Gr-1 (BDPharMingen) followed by phycoerythrin conjugated streptavidin (Immunotech, Portland, ME, USA). Human IL-8 was detected in $1 \%$ paraformaldehyde fixed frozen sections using biotinylated goat antihuman IL-8 (R\&D Systems, Minneapolis, MN, USA) followed by streptavidin-peroxidase and FITC conjugated tyramide (Perkin Elmer Life Sciences). YoPro-3 (Molecular Probes, Eugene, OR, USA) was used as a nuclear counterstain in some experiments. Fluorescence images were acquired using a Zeiss LSM510 confocal microscope.

\section{Transmission electron microscopy}

Tissue specimens were washed with cold PBS, cut into small pieces, and fixed immediately in $2.5 \%$ glutaraldehyde in phosphate buffer $\mathrm{pH}$ 7.2. After fixation, tissue was washed in buffer, dehydrated, and embedded in Epon. Ultrathin sections were cut with diamond knives on an ultramicrotome and mounted on uncoated mesh grids. Sections were contrasted with uranyl acetate and lead citrate and examined under a Philips CM10 electron microscope.

\section{Stool water content measurement}

Stool water content was calculated by a previously described method. ${ }^{23}$ In brief, caecal stool samples and faecal pellets were collected in tubes. The water content of samples was 

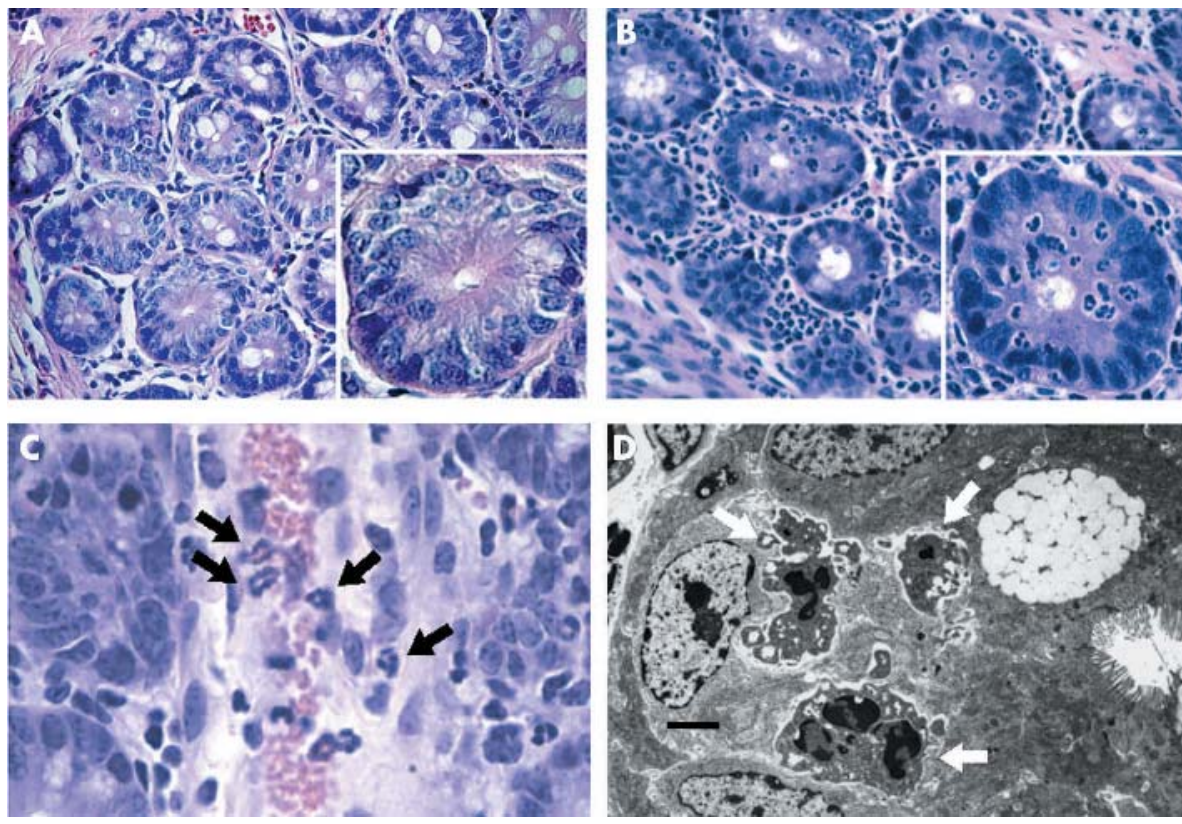

Figure 3 Microscopic features of neutrophil infiltrates in caecum of FABPrtTA $\times$ TRE2IL-8 mice after DOX induction. (A) (B) H\&E stained sections of caecum from FABP-rtTA $\times$ TRE2IL-8 mice were prepared in uninduced mice (A) and 48 hours following DOX induction (B). The main images are at $200 \times$ magnification and the single crypts in the inset at $600 \times$. A dense infiltrate of neutrophils is observed in the lamina propria and intestinal epithelium following DOX induction, but neutrophils did not migrate into the crypt lumen and epithelial cellular damage was not observed. (C) Neutrophils (black arrows) are undergoing diadepedesis through postcapillary venules in the lamina propria after DOX induction $(600 \times)$. (D) Transmission electron microscopy images in DOX-induced FABP-rtTA $\times$ TRE2IL8 mice show neutrophils with intact granules (white arrows) in the paracellular space (scale bar $=1 \mu \mathrm{m}$ ). calculated from the wet weight and the dry weight measured after dessication of the stool sample in a heating block.

Ussing chamber analysis of intestinal tissue Small segments of caecal tissue were surgically isolated from anesthesized mice, washed to remove the lumenal contents, placed into P2300 Ussing chambers (Physiological Instruments, San Diego, CA, USA), and allowed to equilibrate with oxygenated $\left(95 \% \mathrm{O}_{2} / 5 \% \mathrm{CO}_{2}\right)$ Krebs buffer at $37^{\circ} \mathrm{C}$ for five minutes. After equilibration, the voltage was clamped at $5 \mathrm{mV}$ using a multichannel voltage/current clamp (Model VCCMC6, Physiological Instruments) and recordings of transmural resistance and short circuit current were taken at five minute intervals or at one minute intervals following simultaneous addition of forskolin (Sigma) to the apical and basolateral chambers to achieve a final concentration of $10 \mu \mathrm{M}$.

Statistical analysis

Differences between the mean values for groups were analysed by Student's $t$ test. A p value of $<0.05$ was considered as significant.

\section{RESULTS}

\section{Development of transgenic mice with tetracycline} regulated expression of human IL-8

In order to generate a model of inducible CXC chemokine expression in the intestinal epithelium of transgenic mice, transgenic mice were first made using a transgene consisting of the human IL-8 cDNA linked to a promoter containing a tetracycline response element (fig 1A). Human IL-8 binds to CXCR2 on mouse neutrophils, eliciting chemotaxis and calcium flux with a potency similar to endogenous mouse chemokines that also bind to mouse CXCR2 ${ }^{24}$ Use of the CDNA for human IL-8 rather than an endogenous mouse CXCR2 binding chemokine for our construct allowed development of a system in which transgene derived chemokine expression could be clearly distinguished from endogenous mouse CXC chemokine expression using immunoassays specific for human IL-8. Primary cell lines were established from neonatal lung tissue of mice representing four independent transgenic lines that had integrated either the
tetOIL-8 or TRE2IL-8 transgenes. The cell lines were transduced with adenoviruses encoding a reverse tetracycline transactivator (rtTA) in the presence and absence of DOX to assay for DOX-induced IL-8 expression (table 1). Cells from one of the TRE2IL-8 transgenic lines tested (\#2) had a much higher level of IL-8 expression than any of the other lines following DOX induction, with a 200 -fold increase of IL-8 in the presence of DOX. This TRE2IL-8 transgenic line was selected for further analysis.

\section{Selection of transgenic mice with intestine specific expression of the reverse tetracycline transactivator} Two types of transgenic mice in which rtTA expression was selectively targeted to intestinal epithelial cells were evaluated for use in combination with the TRE2IL-8 mice to generate an intestine specific inducible IL- 8 mouse model. In the FABP-rtTA mice, ${ }^{20}$ the rtTA transgene is under the control of a modified liver fatty acid binding protein promoter (Fabpl $l^{4 \mathrm{X}}$ at -132 promoter) in which four extra copies of a 35 bp element important for colonic expression were inserted just upstream of the endogenous location of this element to boost the activity of the promoter in the colon. ${ }^{25}$ We also attempted to develop transgenic mice with rtTA expression throughout the colon using the human downregulated in adenoma (DRA) promoter. The intestinal expression of mRNA for rtTA was compared in FABP-rtTA mice and one line of DRA-rtTA mice by RNase protection assay (fig 1B). Intestinal tissue from the FABP-rtTA mice had a much higher level of rtTA mRNA than tissue from the DRA-rtTA transgenic mice. The highest levels of expression of the FABP-rtTA transgene were detected in distal ileum and the caecum in agreement with previous analysis of the functional activity of this rtTA transgene in different tissues. ${ }^{20}$ To assess the relative expression levels of the FABP-rtTA transgene in different regions of the mouse intestine, double transgenic mice were bred that had the FABP-rtTA transgene and a tetracycline regulated luciferase reporter transgene (tetOluc mice). Individual tissues from FABP-rtTA $x$ tetOluc mice were harvested three days after DOX induction and homogenised for measurement of luciferase activity. Induction of luciferase activity was restricted to the ileum, caecum, and proximal colon (fig $\mathrm{lC}$ ). 
Induction of intestinal IL-8 expression in double transgenic mice

We next bred mice that were double transgenic for the FABPrtTA and TRE2IL- 8 transgenes. These mice were induced with DOX for three days and the levels of IL-8 expression analysed in serum and tissue homogenates by ELISA. DOX induction resulted in over 60 -fold induction of IL- 8 expression at the protein level in the caecum and strong induction in the ileum and proximal colon (table 2 ).

Analysis of the kinetics of appearance of IL-8 in the intestine following DOX induction showed that the increase in IL-8 was already apparent at 24 hours and the IL-8 concentration continued to increase between 24 and 72 hours (fig 2). We also assessed the effect of DOX induction on the plasma concentration of IL-8 (table 3). Mice with just the TRE2IL- 8 transgene had a low basal plasma level of IL- 8 that was not affected by the administration of DOX (data not shown). DOX induction for five days in double transgenic mice resulted in a 6.9-fold induction in plasma IL-8 (table 3 ).

\section{IL-8 induction in the caecum and proximal colon} leads to neutrophil infiltration

We next examined the phenotypic consequences of IL-8 induction by DOX in the double transgenic mice by histology. DOX induction was associated with appearance of a dense infiltrate of Gr-1 positive neutrophils in the caecum and large intestine by 48 hours after DOX induction (fig 3A, B). However, no other histological abnormalities were detected. Some of the neutrophils in the DOX induced mice were concentrated around blood vessels within the intestinal
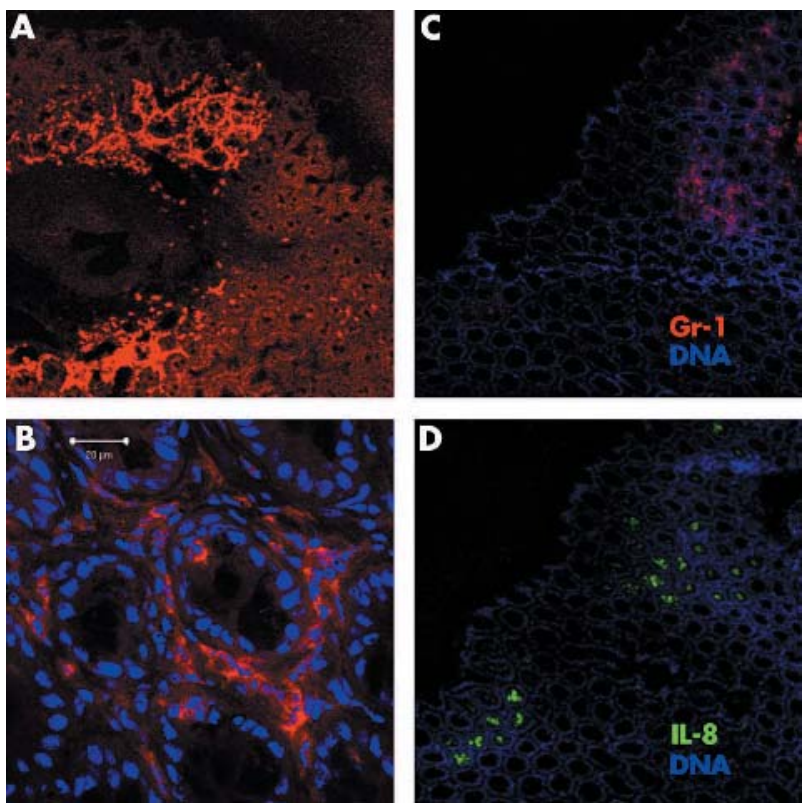

Figure 4 Neutrophil infiltration in caecum of DOX-induced FABP-rtTA $\times$ TRE2IL-8 mice is localised to discrete patches. (A) Biotin-conjugated antibody to Gr-1 followed by streptavidin-phycoerythrin was used to localise neutrophils $(\mathrm{red})$ in frozen sections of FABP-rTTA $\times$ TRE2IL- 8 mice induced with DOX for five days (50x magnification) and counterstained with YoPro-3 (pseudocoloured blue). Areas of intense neutrophil infiltration are separated by groups of crypts in which no neutrophils are detected. A similar patchy pattern of neutrophil infiltration was observed in sections from four other double transgenic mice stained with the same antibodies. (B) High power $(600 \times)$ image of a crypt demonstrates $\mathrm{Gr}-1+$ neutrophils that have migrated into the paracellular space between epithelial cells. (C) (D) Consecutive sections of caecum from five day induced double transgenic mice were stained for Gr-1 (C) and IL-8 (D) showing that Gr-1+ cells are present in the same patches where IL-8 is expressed.

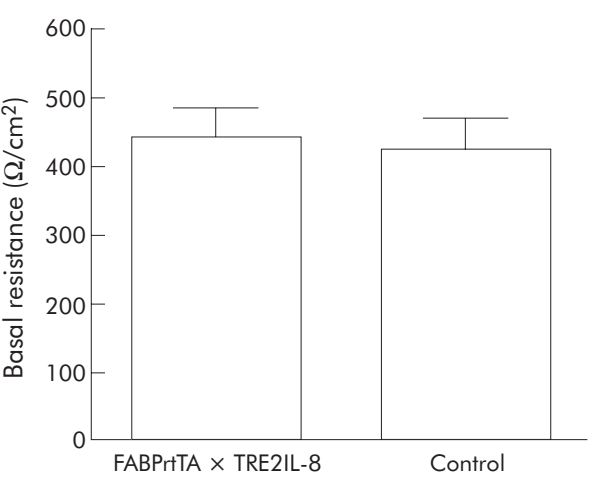

Figure 5 DOX induction of FABP-rtTA $\times$ TRE2IL-8 mice does not change the basal transmural resistance of the caecum. Double transgenic mice and controls ( $n=4$ for each group) were induced for five days by DOX supplementation of the drinking water. Caecal tissue from the mice was mounted in Ussing chambers for measurement of the basal transmural resistance, which is reported as the mean (SD). The difference between the groups was not statistically significant $(p>0.05)$. Similar results were obtained in a second experiment with groups of six mice.

submucosa, suggesting the IL-8 elicited exit of neutrophils from the bloodstream might be initiated at postcapillary venules found in the intestinal wall. Few if any Gr-1 positive cells were identified in control mice.

Three features of the observed neutrophil infiltrates were of particular interest. Firstly, examination of many sections of caecum from induced double transgenic mice revealed that even when the recruited neutrophils broached the basement membrane of a crypt, the neutrophils remained confined to the paracellular space below the tight junctions and were never observed to reach the crypt lumen to yield a lesion comparable to a crypt abscess (fig 3A, B). The induced IL-8 gradient was never sufficient to effect complete transepithelial migration of the neutrophils, even if DOX induction was continued for several weeks (data not shown). Secondly, even the densest neutrophil infiltrates observed did not result in histologically recognisable epithelial injury or ulceration. One contributing factor to the absence of epithelial damage by the infiltrating neutrophils may be limited degranulation of the recruited neutrophils. Transmission electron micrographs of neutrophils located within crypts revealed that the majority of the granules identified within the neutrophil cytoplasm were intact without evidence of degranulation (fig 3D and data not shown). A third feature of the neutrophil infiltrate best revealed by the immunofluorescence studies was its patchy distribution within the lamina propria (fig 4A, B). One potential explanation of this pattern of infiltration was that IL-8 was only expressed by epithelial cells derived from stem cells in a subset of intestinal crypts. Immunostaining of consecutive sections with antibodies to IL-8 and Gr-1 revealed that neutrophil infiltration was localised to the same areas where IL- 8 expression was detected (fig 4C, D).

Neutrophil infiltration induced by DOX in FABP-rtTA $\times$ TRE2IL- 8 mice is associated with an increase in stool water content

We used several experimental approaches to investigate whether IL-8 induced neutrophil recruitment to the intestinal mucosa could elicit any functional abnormalities in the epithelium. One parameter that was examined was the water content of the stool. We hypothesised that the influx of neutrophils might result in the release of mediators that would provoke epithelial cell ion secretion leading to net water movement into the lumenal space. A statistically significant increase in the water content of both the caecal 
Table 4 Increased stool water content in FABP-rtTA $x$ TRE2IL-8 mice after DOX treatment ${ }^{*}$

\begin{tabular}{lll}
\hline & \multicolumn{2}{l}{ Stool water content $\left(\% \mathrm{H}_{2} \mathrm{O}\right.$; mean $\left.(\mathrm{SD})\right)$} \\
\cline { 2 - 3 } Source of stool & Control mice & FABP-rtTA $\times$ TRE2IL-8 mice \\
\hline Caecum & $80.6(1.5)$ & $84.7(1.7) \dagger$ \\
Distal colon & $60.1(0.8)$ & $69.9(2.5) \ddagger$ \\
\hline
\end{tabular}

*Three days after initiating DOX treatment, stool samples were recovered from the caecum or distal colon of control and FABP-rtTA $\times$ TRE2IL-8 mice ( $n=4$ for both groups) for determination of wet and dry stool weight. The mean stool water content was calculated for the two groups of mice and compared by Student's $t$ test.

tThe $p$ value for the difference in water content between the caecal samples was 0.012 . Significant increases in the cecal stool water content were observed in two additional experiments with groups of 4-6 mice. \#For the distal colon samples, the $p$ value was 0.001 .

and colonic stool was observed in double transgenic mice after DOX induction (table 4). To determine if the increased water content was associated with other perturbations in epithelial ion transport and resistance, Ussing chamber experiments were performed. The mean transmural electrical resistance of the caecal tissue recovered from DOX induced double transgenic mice was comparable to the value for DOX treated control mice (fig 5). Basal short circuit current and the change in short circuit current elicited by forskolin stimulation were also not significantly altered (data not shown). In addition, the in vivo permeability of the intestine to orally ingested FITC dextran (molecular weight of $4000 \mathrm{kDa})$ in double transgenic and control mice 24 hours after DOX induction was not statistically different (data not shown).

\section{DISCUSSION}

The directed migration of leukocytes is controlled by multiple classes of chemotactic factors including chemokines, leukotrienes, complement derived anaphylatoxins, eicosanoids, and bacterially derived peptides such as fMLP. Identifying the specific contributions made by an individual species of chemotactic factor in vivo represents a substantial experimental challenge. We developed a novel mouse model featuring DOX inducible transgenic expression of a CXCR2 binding chemokine by intestinal epithelial cells in order to determine whether a CXC chemokine gradient alone is sufficient to elicit complete transepithelial migration of neutrophils. This in vivo model demonstrates that acute induction of the human CXC chemokine IL- 8 restricted to mouse intestinal epithelial cells is sufficient to efficiently trigger extravasation of neutrophils from the vasculature, accumulation of neutrophils within the lamina propria, and penetration by the neutrophils through the epithelial basement membrane to reside in the paracellular space beneath the tight junction. However, the induced chemokine gradient does not result in full activation of the recruited neutrophils and the recruited neutrophils fail to breach the tight junctions to reach the intestinal lumen. Based on these results, we hypothesise that CXCR2 ligands such as IL-8 that are to be predominantly released from the basolateral side of polarised epithelial cells are competent to elicit neutrophil recruitment into the lamina propria, but require the concurrent presence of additional chemotactic and/or activating signals to effect complete transepithelial migration and to fully activate the recruited neutrophils.

The inducible transgenic mouse model of chemokine expression described in this report is the first model in which administration of the inducing agent (DOX in this system) into the intact transgenic animal results in directed leukocyte migration that is not observed before induction. A series of chemokine transgenic mouse models have been described previously..$^{14-16}{ }^{26-30}$ In some of these mouse models, constitutive expression of a chemokine from a tissue specific promoter resulted in selective recruitment of certain leukocyte populations responsive to the chemokine to the site of expression. ${ }^{16}{ }^{26-29}$ However, in other models strong constitutive expression of a chemokine resulted in desensitisation and hyporesponsiveness of leukocytes to the chemokine, even if it is expressed in a tissue specific pattern. ${ }^{14}{ }^{15} 27$ This may be explained by the fact that leukocyte migration depends on a gradient of the attracting chemokine and high constitutive chemokine expression can lead to leukocyte desensitisation and inability to migrate. In two models featuring constitutive human IL-8 expression, ${ }^{14}{ }^{15}$ desensitisation of neutrophils to the effects of IL- 8 resulted in peripheral neutrophilia and decreased CD62L expression by the blood neutrophils. These neutrophil changes were not observed in our model (data not shown) despite the presence of a low basal level of circulating IL-8, consistent with our observations that DOX induction always elicited directed migration of neutrophils from blood into the intestinal lamina propria. One previous model also used a tetracycline regulated system to control expression of the mouse CXC chemokine KC..$^{30}$ DOX induction of these double transgenic mice did not result in foci of neutrophil infiltration, perhaps because induced chemokine expression in almost all tissues prevented establishment of a chemokine gradient that could attract neutrophils.

One characteristic feature of the inducible IL- 8 expression mouse model is the patchy distribution of the neutrophil infiltrates in the caecum and colon that corresponded to the areas of IL-8 expression. Variegated expression of transgenes is known to be a common occurrence when tandem arrays containing a transgene sequence randomly integrate into genomic DNA. ${ }^{31}{ }^{32}$ The most likely explanation of the patchy neutrophil infiltrates in the present model is epigenetic silencing of either the FABP-rtTA transgene, the TRE2IL-8 transgene, or both transgenes in intestinal epithelial cells in which the Fabpl $l^{4 \mathrm{X}}$ at -132 promoter is normally active.

The inducible model for IL-8 expression described in this report proved useful for analysis of the functional consequences of inducible IL-8 expression despite the restriction of the neutrophil infiltration to localised areas in the caecum. The increased water content of the caecal and colonic stool in the induced double transgenic mice after three days of DOX induction demonstrates that the recruited neutrophils result in a local perturbation of epithelial cell water transport leading to an increased water content. While DOX was the only tetracycline used in this study to induce IL-8 expression, 4-epidoxycyline is an alternative DOX derivative that also efficiently induces rtTA activity and has the advantage of not exerting an antibiotic effect on the intestinal bacterial flora. ${ }^{33}$

One of the more striking characteristics of the neutrophil infiltrates induced by DOX in the caecum of double transgenic mice was the presence of minimal tissue injury and the absence of complete transepithelial migration of neutrophils. As acute bacterial infection at a mucosal site resulting in neutrophil infiltration typically results in complete transepithelial migration of neutrophils and is accompanied by tissue injury, we conclude that other chemotactic and activating factors for neutrophils are needed to bring about this "complete" neutrophil response. Along these lines, studies of chemotactic factors elicited by infection of model intestinal epithelia with Salmonella have identified basolaterally secreted chemokines as well as an apically secreted molecule originally designated PEEC for pathogen elicited epithelial chemoattractant. ${ }^{34}{ }^{35}$ Purification and biochemical characterisation of PEEC has recently identified this material as the eicosanoid hepoxilin $\mathrm{A}_{3} \cdot{ }^{36}$ Based on the absence of complete transepithelial migration in our model, 
we speculate that apically secreted chemotactic factors derived from the epithelial cells such as hepoxilin $A_{3}$ are also necessary to drive neutrophils across epithelial tight junctions to reach the lumenal space. While the chemokine IL-8 has been shown previously to elicit degranulation of human neutrophils, ${ }^{37} 38$ most of the granules in the neutrophils infiltrating intestinal tissue in the double transgenic mouse model appeared to be intact as assessed by transmission electron microscopy. We speculate that more efficient degranulation of mouse neutrophils recruited by CXCR2 binding chemokines may require additional activating stimuli. Interestingly, recruitment of leukocytes without concurrent inflammatory activation has been observed in other chemokine overexpressing transgenic mice. ${ }^{26}{ }^{29}$ Another explanation of the absence of degranulation in the inducible IL-8 mice is that IL-8 elicited degranulation of human neutrophils may be primarily a function of the CXCRI receptor found only on human neutrophils rather than the CXCR2 receptor shared between mouse and human neutrophils. ${ }^{39}$

Future studies on this inducible mouse model of intestinal IL-8 expression and neutrophil infiltration will be focused in several areas. As several small molecule CXCR2 antagonists have shown inhibitory activity in other animal models of chemokine induced inflammation ${ }^{40-42}$ this model provides an ideal in vivo system to assess the activity of candidate drugs that act by interfering with CXC chemokine binding to CXCR2. Secondly, these mice represent an excellent in vivo model in which to characterise how neutrophil infiltration modifies epithelial gene expression since the inflammatory infiltrate is restricted to the intestinal epithelium. Finally, the intense neutrophil infiltrate achieved by use of a tissue specific promoter for rtTA provides proof of principle that similar models of inducible, localised neutrophil infiltration can be created by using other lines of rtTA transgenic mice in which promoters with different patterns of tissue specific expression are substituted for the Fabpl ${ }^{4 \mathrm{X}}$ at -132 promoter used in this study.

\section{ACKNOWLEDGEMENTS}

This work was supported by grants from the German Research Foundation (Deutsche Forschungsgemeinschaft; Ku 1328/1-1 to TK, Lu 816/1-1 to A.L.), the German Ministry for Education and Technology (German IBD network; BMBF 01GI0286), the Crohn's and Colitis Foundation of America (Senior Research Award to IRW), and the National Institutes of Health (DK-65345 to MEE, DK-55850 to TRZ, DK-61941 to DM, and DK-64399 supporting the Imaging Core Facility of the Emory Digestive Diseases Research Development Center). We thank Dr David Martin for his assistance in making the TRE2IL-8 transgenic mice and Dr Gary Wu for providing the $3.6 \mathrm{~kb}$ DRA promoter used to make the DRA-rtTA construct. The FABP-rtTA transgenic mice were the kind gift of Dr Jeffrey I Gordon (Washington University School of Medicine, St Louis, MO, USA). The tetOluc mice were originally made by Dr Hermann Bujard's laboratory at the University of Heidelberg and were provided by Dr Hans Winkler (Beth Israel Deaconess Medical Center, Boston, MA, USA). The pUC-IL-8 plasmid was kindly provided by Dr John Ansel.

\section{Authors' affiliations \\ T Kucharzik, J T Hudson III, A Lügering, J A Abbas, M Bettini, J G Lake, I R Williams, Department of Pathology and Laboratory Medicine, Epithelial Pathobiology Research Unit, Emory University School of Medicine, Atlanta, GA, USA \\ T Kucharzik, A Lügering, Department of Medicine B, University of Münster, Germany \\ M E Evans, T R Ziegler, D Merlin, Department of Medicine, Division of Digestive Diseases, Emory University School of Medicine, Atlanta, GA, USA \\ J L Madara, Pritzker School of Medicine, University of Chicago, Chicago, IL, USA \\ Conflict of interest: none declared.}

\section{REFERENCES}

1 McCormick BA, Colgan SP, Delp-Archer C, et al. Salmonella typhimurium attachment to human intestinal epithelial monolayers: transcellular signalling to subepithelial neutrophils. J Cell Biol 1993;123:895-907.

2 Eckmann L, Kagnoff MF, Fierer J. Epithelial cells secrete the chemokine interleukin-8 in response to bacterial entry. Infect Immun 1993;61:4569-74.

3 McCormick BA, Hofman PM, Kim J, et al. Surface attachment of Salmonella typhimurium to intestinal epithelia imprints the subepithelial matrix with gradients chemotactic for neutrophils. J Cell Biol 1995;131:1599-608.

4 Eckmann L, Jung HC, Schurer-Maly C, et al. Differential cytokine expression by human intestinal epithelial cell lines: regulated expression of interleukin 8. Gastroenterology 1993;105:1689-97.

5 Izzo RS, Witkon K, Chen Al, et al. Interleukin-8 and neutrophil markers in colonic mucosa from patients with ulcerative colitis. Am J Gastroenterol 1992:87: 1447-52

6 Mazzucchelli L, Hauser C, Zgraggen K, et al. Expression of interleukin-8 gene in inflammatory bowel disease is related to the histological grade of active inflammation. Am J Pathol 1994; 144:997-1007.

7 Nickoloff BJ, Karabin GD, Barker JN, et al. Cellular localization of interleukin8 and its inducer, tumor necrosis factor-a in psoriasis. Am J Pathol $1991 ; 138: 129-40$.

8 Madara JL, Nash S, Parkos C. Neutrophil-epithelial cell interactions in the intestine. Adv Exp Med Biol 1991;314:329-34.

9 Godaly G, Proudfoot AE, Offord RE, et al. Role of epithelial interleukin-8 (IL-8) and neutrophil IL-8 receptor A in Escherichia coli-induced transuroepithelial neutrophil migration. Infect Immun 1997;65:3451-6.

10 Matsumoto T, Yokoi K, Mukaida N, et al. Pivotal role of interleukin-8 in the acute respiratory distress syndrome and cerebral reperfusion injury. J Leukoc Biol 1997;62:581-7.

11 Harada A, Kuno K, Nomura $\mathrm{H}$, et al. Cloning of a cDNA encoding a mouse homolog of the interleukin-8 receptor. Gene 1994;142:297-300.

12 Cacalano G, Lee J, Kikly K, et al. Neutrophil and B cell expansion in mice that lack the murine IL-8 receptor homolog. Science 1994;265:682-4.

13 Frendeus B, Godaly G, Hang L, et al. Interleukin-8 receptor deficiency confers susceptibility to acute pyelonephritis. J Infect Dis 2001;183(Suppl 1):S56-60.

14 Simonet WS, Hughes TM, Nguyen $\mathrm{HQ}$, et al. Long-term impaired neutrophil migration in mice overexpressing human interleukin-8. J Clin Invest 1994;94:1310-19.

15 Horiguchi $\mathbf{H}$, Harada A, Oguma $\mathrm{E}$, et al. Cadmium-induced acute hepatic injury is exacerbated in human interleukin-8 transgenic mice. Toxicol Appl Pharmacol 2000;163:231-9.

16 Ohtsuka Y, Lee J, Stamm DS, et al. MIP-2 secreted by epithelial cells increases neutrophil and lymphocyte recruitment in the mouse intestine. Gut 2001:49:526-33.

17 Gossen M, Freundlieb S, Bender G, et al. Transcriptional activation by tetracyclines in mammalian cells. Science 1995;268:1766-9.

18 Matsushima K, Morishta K, Yoshimura T, et al. Molecular cloning of a human monocyte-derived neutrophil chemotactic factor (MDNCF) and the induction of MDNCF mRNA by interleukin 1 and tumor necrosis factor. J Exp Med 1988; 167:1883-93.

19 Gossen M, Bujard H. Tight control of gene expression in mammalian cells by tetracycline-responsive promoeters. Proc Natl Acad Sci U S A 1992;89:5547-51

20 Saam JR, Gordon JI. Inducible gene knockouts in the small intestinal and colonic epithelium. J Biol Chem 1999;274:38071-82.

21 Furth PA, St. Onge L, Boger H, et al. Temporal control of gene expression in transgenic mice by a tetracycline-responsive promoter. Proc Natl Acad Sci U S A 1994;91:9302-6.

22 Baron U, Schnappinger D, Helbl V, et al. Generation of conditional mutants in higher eukaryotes by switching between the expression of two genes. Proc Natl Acad Sci U S A 1999;96:1013-18.

23 Wang KS, Ma T, Filiz F, et al. Colon water transport in transgenic mice lacking aquaporin-4 water channels. Am J Physiol Gastrointest Liver Physiol 2000;279:G463-G470.

24 Rot A. Chemotactic potency of recombinant human neutrophil attractant/ activation protein-1 (interleukin-8) for polymorphonuclear leukocytes of different species. Cytokine 1991;3:21-7.

25 Simon TC, Cho A, Tso P, et al. Suppressor and activator functions mediated by a repeated heptad sequence in the liver fatty acid-binding protein gene (Fabpl). Effects on renal, small intestinal, and colonic epithelial cell gene expression in transgenic mice. J Biol Chem 1997;272:10652-63.

26 Lira SA, Zalamea P, Heinrich JN, et al. Expression of the chemokine N51/KC in the thymus and epidermis of transgenic mice results in marked infiltration of a single class of inflammatory cells. J Exp Med 1994;180:2039-48.

27 Rutledge BJ, Rayburn H, Rosenberg R, et al. High level monocyte chemoattractant protein-1 expression in transgenic mice increases their susceptibility to intracellular pathogens. J Immunol 1995;155:4838-43.

28 Tani $M$, Fuentes $M E$, Peterson JW, et al. Neutrophil infiltration, glial reaction, and neurological disease in transgenic mice expressing the chemokine N51/ KC in oligodendrocytes. J Clin Invest 1996;98:529-39.

29 Gunn MD, Nelken NA, Liao X, et al. Monocyte chemoattractant protein-1 is sufficient for the chemotaxis of monocytes and lymphocytes in transgenic mice but requires an additional stimulus for inflammatory activation. J Immunol 1997; 158:376-83.

30 Wiekowski MT, Chen SC, Zalamea P, et al. Disruption of neutrophil migration in a conditional transgenic model: evidence for CXCR2 desensitization in vivo. $\mathrm{J}$ Immunol 2001;167:7102-10.

31 Martin DI, Whitelaw E. The vagaries of variegating transgenes. Bioessays 1996;18:919-23. 
32 Opsahl ML, McClenaghan M, Springbett A, et al. Multiple effects of genetic background on variegated transgene expression in mice. Genetics 2002; 160:1107-12.

33 Eger K, Hermes M, Uhlemann K, et al. 4-Epidoxycyline: an alternative to doxycycline to control gene expression in conditional mouse models. Biochem Biophys Res Commun 2004;323:979-86.

34 Gewirtz AT, McCormick B, Neish AS, et al. Pathogen-induced chemokine secretion from model intestinal epithelium is inhibited by lipoxin A4 analogs. J Clin Invest 1998;101:1860-9.

35 McCormick BA, Parkos CA, Colgan SP, et al. Apical secretion of a pathogenelicited epithelial chemoattractant activity in response to surface colonization of intestinal epithelia by Salmonella typhimurium. J Immunol 1998; 160:455-66.

36 Mrsny RJ, Gewirtz AT, Siccardi D, et al. Identification of hepoxilin A3 in inflammatory events: A required role in neutrophil migration across intestinal epithelia. Proc Natl Acad Sci U S A 2004;101:7421-6.
37 Smith RJ, Sam LM, Leach KL, et al. Postreceptor events associated w ith human neutrophil activation by interleukin-8. J Leukoc Biol 1992;52:17-26.

38 Barlic J, Andrews JD, Kelvin AA, et al. Regulation of tyrosine kinase activation and granule release through $\beta$-arrestin by CXCR1. Nat Immunol 2000; 1:227-33.

39 Murphy PM. Neutrophil receptors for interleukin-8 and related CXC chemokines. Semin Hematol 1997;34:311-18.

40 White JR, Lee JM, Young PR, et al. Identification of a potent, selective nonpeptide CXCR2 antagonist that inhibits interleukin-8-induced neutrophil migration. J Biol Chem 1998;273:10095-8.

41 Podolin PL, Bolognese BJ, Foley JJ, et al. A potent and selective nonpeptide antagonist of CXCR2 inhibits acute and chronic models of arthritis in the rabbit. J Immunol 2002; 169:6435-44.

42 Hay DW, Sarau HM. Interleukin-8 receptor antagonists in pulmonary diseases. Curr Opin Pharmacol 2001;1:242-7.

\section{EDITOR'S QUIZ: GI SNAPSHOT}

\section{Hypoalbuminaemia and gastric mass}

\section{Clinical presentation}

A 45 year old woman presented with longstanding dyspepsia associated with oedema and a $4 \mathrm{~kg}$ weight loss over the previous six months. She gave no history of haematemesis or blood in stool, nocturnal sweating, or chronic diarrhoea. There was no familial history of gastric carcinoma. Surgical history included cure of eventration. Physical examination revealed tenderness in the epigastrium. Routine laboratory investigations revealed: haemoglobin $9.6 \mathrm{~g} \%$ (normal range 10-14), mean corpuscular volume $80 \mathrm{fl}$ (normal range 7595 ), serum albumin $25 \mathrm{~g} / \mathrm{l}$ (normal range 35-50), C reactive protein $<3$ (normal range $<10$ ), lactate dehydrogenase $355 \mathrm{IU}$ (normal range $<600$ ), and $\beta_{2}$ microglobulin 2 (normal range 0.5-1.2). The patient underwent upper gastrointestinal endoscopy with gastric biopsy and endoscopic ultrasound of the stomach (fig 1-3).

\section{Question}

What is the diagnosis? What other entities could present with this endoscopic and histological picture?

See page 1589 for answer

This case is submitted by:

K Honein, J Boujaoude

Department of Gastroenterology, Hotel-Dieu de France Hospital, Saint Joseph University, Lebanon

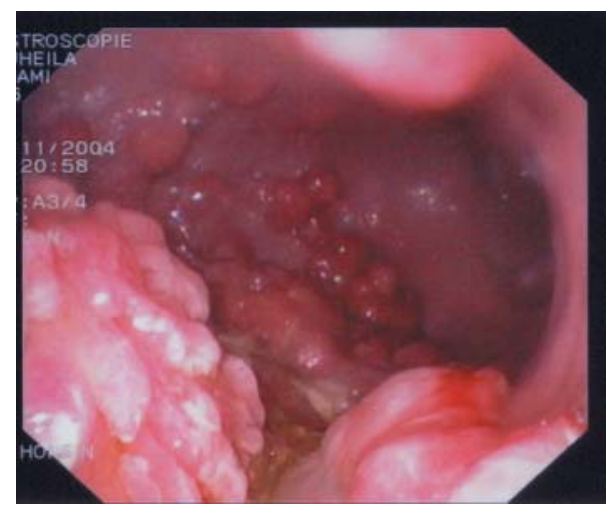

Figure 1 Upper gastrointestinal endoscopy.

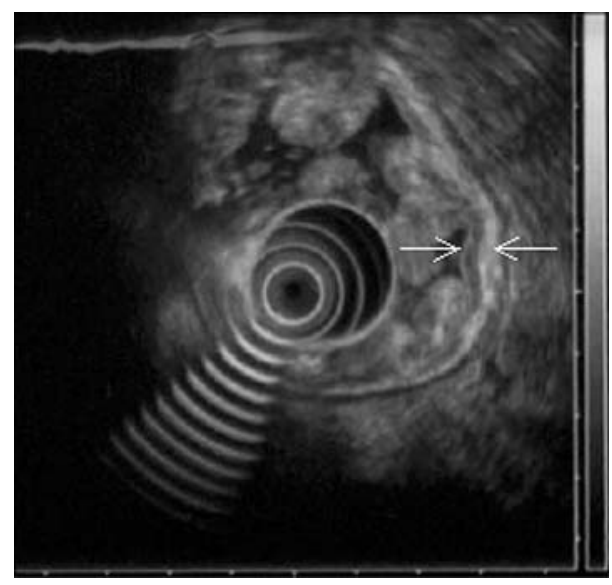

Figure 2 Endoscopic ultrasound of the stomach.

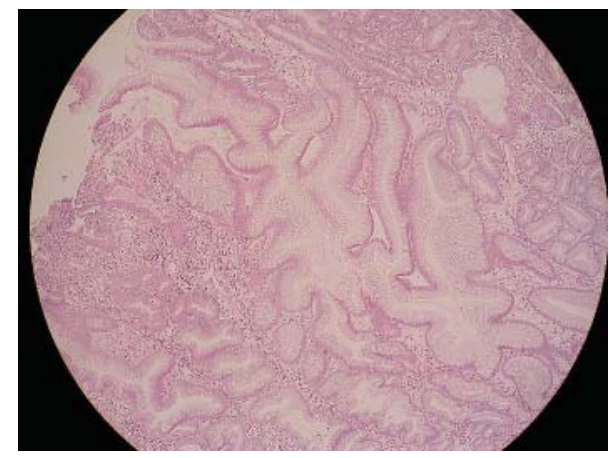

Figure 3 Full thickness gastric mucosal biopsy.

C Ghora

Department of Pathology, Hotel-Dieu de France Hospital, Saint Joseph University, Lebanon

Correspondence to: Professor J Boujaoude, Department of Gastroenterology, Hotel-Dieu de France Hospital, Saint Joseph University, Beyrouth 961, Lebanon; jbouj@hotmail.com

doi: $10.1136 /$ gut.2005.071183 